\title{
Zu oft diagnostiziert
}

Fragestellung: Bei wie viel Prozent der Frauen mit Übergewicht und Kopfschmerzen liegt tatsächlich ein Pseudotumor cerebri vor?

Hintergrund: Bei der idiopathischen intrakraniellen Hypertension handelt es sich um eine Erkrankung mit erhöhtem Hirndruck bei übergewichtigen jungen Frauen in Kombination mit Stauungspapillen. In der Kernspintomografie finden sich häufig zusätzliche Zeichen wie beispielsweise eine Empty-Sella oder verdickte Nervi optici. Die Diagnose setzt neben den klinischen Symptomen den Nachweis einer Stauungspapille und einen erhöhten Liquordruck voraus, in Kombination mit dem Ausschluss anderer Ursachen wie beispielsweise einer Sinusvenenthrombose.

Patienten und Methodik: Es handelt sich um eine monozentrische Studie einer großen Abteilung für Neuroophthalmologie in den USA. Erfasst wurden Patientinnen, die wegen der Diagnose idiopathische intrakranielle Hypertension in einem Zeitraum von acht Monaten überwiesen wurden. Die Autoren erfassten die Zahl der Fehldiagnosen und die Gründe für die entsprechende falsche diagnostische Einordnung.

Ergebnisse: Im Zeitraum zwischen November 2013 und Juni 2014 wurden 1.249 Patientinnen untersucht, 165 wurden überwiesen mit der Verdachtsdiagnose idiopathische intrakranielle Hypertension. Bei 86 Patientinnen hatte der überweisende Arzt bereits die Diagnose gestellt. Nach gründlicher Untersuchung ließ sich die Diagnose bei 34 dieser Patientinnen nicht bestätigen, was einer Fehlerrate von $39,5 \%$ entspricht. 79 Patientinnen wurden überwiesen, um einen Pseudotumor cerebri zu bestätigen oder auszuschließen. Von diesen hatten $81 \%$ keinen Pseudotumor cerebri. Der häufigste Grund für die Fehldiagnose war eine vermutete Stauungspapille, die aber andere Ursachen wie beispielsweise Drusenpapille hatte. Bei einem Teil der Patienten war bei vorbestehenden chronischen Kopfschmerzen und Übergewicht keine Liquordruckmessung erfolgt. Erschreckend ist, dass viele der Patienten, bei denen sich die Diagnose nicht bestätigen ließ, bereits zum Teil aggressiv behandelt worden waren, darunter ein Patient mit einer Liquordrainage und vier, die zu einem operativen Eingriff überwiesen worden waren.

Fisayo A, Bruce BB, Newman $\mathrm{NJ}$ et al. Overdiagnosis of idiopathic intracranial hypertension. Neurology 2016; 86: 341 - 50
Schlussfolgerungen: Bei bis zu $40 \%$ der Patientinnen, die mit der Diagnose idiopathische intrakranielle Hypertension in eine Neuroophthalmologie überwiesen werden, lässt sich die Diagnose nicht bestätigen.

\section{- Kommentar von Hans-Christoph Diener, Essen}

\section{Diagnose gründlich abklären}

Diese monozentrische, retrospektive Studie ist sehr lehrreich, da sie zeigt, dass die Kombination von chronischen Kopfschmerzen, weiblichem Geschlecht, Übergewicht und Sehstörungen nicht automatisch mit der Diagnose eines Pseudotumors cerebri vereinbar ist. Zunächst muss durch eine gründliche ophthalmologische Untersuchung geklärt werden, ob tatsächlich Stauungspapillen vorliegen. Die Diagnose stützt sich darüber hinaus auf den gemessenen Liquordruck, wobei die derzeit in der Literatur festgelegten Grenzen von 25-30 cm Wassersäule relativ willkürlich und durch prospektive Studien nicht gut belegt sind. Eine medikamentöse Therapie oder wiederholte Liquorpunktionen sind nur dann angezeigt, wenn die Diagnose eines Pseudotumors cerebri bestätigt ist. 